\title{
THE
}

\section{Automatic identification of the number of food items in a meal using clustering techniques based on the monitoring of swallowing and chewing}

\author{
Paulo Lopez-Meyer \\ Stephanie Schuckers \\ Oleksandr Makeyev \\ University of Rhode Island \\ Juan M. Fontana \\ Edward Sazonov
}

Follow this and additional works at: https://digitalcommons.uri.edu/ele_facpubs

This is a pre-publication author manuscript of the final, published article.

Creative Commons License

c) (i) $\Theta \Theta$

This work is licensed under a Creative Commons Attribution-Noncommercial-No Derivative Works 4.0 License.

\begin{abstract}
Citation/Publisher Attribution
Lopez-Meyer, P., Schuckers, S., Makeyev, O., Fontana, J. M., \& Sazonov, E. (2012). Automatic identification of the number of food items in a meal using clustering techniques based on the monitoring of swallowing and chewing. Biomedical Signal Processing and Control, 7(5), 474-480. doi: 10.1016/j.bspc.2011.11.004 Available at: https://doi.org/10.1016/j.bspc.2011.11.004
\end{abstract}

This Article is brought to you for free and open access by the Department of Electrical, Computer, and Biomedical Engineering at DigitalCommons@URI. It has been accepted for inclusion in Department of Electrical, Computer, and Biomedical Engineering Faculty Publications by an authorized administrator of DigitalCommons@URI. For more information, please contact digitalcommons-group@uri.edu. 


\title{
Automatic identification of the number of food items in a meal using clustering techniques based on the monitoring of swallowing and chewing
}

\author{
Paulo Lopez-Meyer ${ }^{a}$, Stephanie Schuckers ${ }^{b}$, Oleksandr Makeyev ${ }^{c}$, Juan M. Fontana ${ }^{a}$, and \\ Edward Sazonova, ${ }^{\text {* }}$ \\ aUniversity of Alabama, Department of Electrical and Computer Engineering, Tuscaloosa, AL \\ 35487, United States \\ ${ }^{\mathrm{b}}$ Clarkson University, Department of Electrical and Computer Engineering, Potsdam, NY 13699, \\ United States \\ cUniversity of Rhode Island, Department of Electrical, Computer, and Biomedical Engineering, \\ Kingston, RI 02881, United States
}

\begin{abstract}
The number of distinct foods consumed in a meal is of significant clinical concern in the study of obesity and other eating disorders. This paper proposes the use of information contained in chewing and swallowing sequences for meal segmentation by food types. Data collected from experiments of 17 volunteers were analyzed using two different clustering techniques. First, an unsupervised clustering technique, Affinity Propagation (AP), was used to automatically identify the number of segments within a meal. Second, performance of the unsupervised AP method was compared to a supervised learning approach based on Agglomerative Hierarchical Clustering (AHC). While the AP method was able to obtain $90 \%$ accuracy in predicting the number of food items, the AHC achieved an accuracy $>95 \%$. Experimental results suggest that the proposed models of automatic meal segmentation may be utilized as part of an integral application for objective Monitoring of Ingestive Behavior in free living conditions.
\end{abstract}

\section{Keywords}

Monitoring of Ingestive Behavior; Food intake; Clustering; Affinity Propagation; Agglomerative Hierarchical Clustering

\section{Introduction}

\begin{abstract}
Dietary self-report methods commonly used for Monitoring of Ingestive Behavior (MIB) like observation, weighed food records, estimated records, diet history food-frequency questionnaires, food recall methods and others have been used intensively in research. However, the majority of them suffer from subject's underreporting (ratio of estimate over actual intake) down to 0.84 [1]. The reason for subject's underreporting related to these methods is mainly due to two main factors: the change in eating behavior that occurs when subjects are asked to record their intake (observation effect), and the subject's tendency to misreport their changed eating behavior (reporting effect) [2-4]. Observation effect can lead
\end{abstract}

*Corresponding author at: Department of Electrical and Computer Engineering, University of Alabama, 101 Houser Hall, Tuscaloosa, AL 35487-0286, United States. Tel.: +1 205348 1981. esazonov@eng.ua.edu (E. Sazonov). 
to an overall decrease of $5 \%$ in intake estimation accuracy, while reporting effect is much more variable and results in decreases ranging from $5 \%$ to $20 \%$, depending on the intake measurement method used [4].

Additionally, underreporting does not only result from a systematical underestimation of portion sizes for all food items, but is also related to avoiding the reporting of certain intake behaviors like snacking [5,6] and consumption of some specific food items [7]. Overall, there is a current need for developing more objective means for MIB that eliminate the burden of subject's active participation in reporting of not only the food size, but also the food periods during the day and different types of food items ingested [7].

In previous studies, we developed a sensor system for non-invasive monitoring of chewing and swallowing and validated the reliability of the produced manual scores [8-10], established a methodology of automatic detection of swallowing instances by acoustical means [11], and proposed and validated several methods for detection and characterization of food intake based on chewing and swallowing $[10,12]$. In this study, we propose a methodology to identify the number of distinct food items within a meal using information extracted from deglutition and mastication patterns. Our methodology is a follow-up on our ongoing research that comprises different stages for automatic objective MIB.

Successful identification of food items not only simplifies understanding of eating behaviors associated with obesity and other eating disorders, but could also be very helpful when monitoring patients with certain diseases. For example, the rate of relapse in patients with anorexia nervosa is estimated to be as high as 50\% [13]. One study showed that the selection of a diet with low energy density and limited variety of food items was associated with a poor treatment outcome [14]. Also, a controlled diet consisting in very-low calorie food items in obese type 2 subjects promotes long-term glycemic control [15]. This study showed that such type of diet produced greater decreases in fasting glucose, with the additional benefit of weight loss. Development of an objective method for monitoring of variety of food items consumed by subjects with diseases like the ones described could potentially lead specialists to a better clinical treatment and recovery control.

Several methodologies have been proposed, urging the need of automatic objective methods for MIB and classification of food items consumed. A study proposed the use of a semiautomatic method to classify the food items from recorded shopping receipts and keep track of their nutritional content to generate suggestions about healthier food items that could help to supplement missing nutrients [16]. However, this method relies in subject's active participation to swipe receipts into a handheld scanner. Also, the shopping suggestions of the system are based on purchases and not necessarily consumed foods. Another study proposed the use of a dining table instrumented with weight sensors and radio frequency identification (RFID) of food containers to detect and distinguish how and what people eat [17]. While this sensor device is still under development, several limitations were mentioned by the authors when reporting their results: external food sources are not allowed different than those contained in RFID containers; weight, nutrition, ingredients of foods, as well as weight and owners of food containers are known a priori; leaning hands and elbows over the table is avoided. These limitations make this device at the moment impractical for freeliving conditions. A different approach was used in another study where multi-sensor based monitoring is used to identify intake gestures, chewing and swallowing to provide timing and food category information [18]. This method successfully discriminates different events by the gesture behavior such as eating with fork and knife, drink from a glass, eat with a spoon, or the use of hands to approach food to the mouth. The motion sensor jacket developed was a research prototype and less complex sensors are being developed. Recently, the use of mobile phones has been proposed in which subjects capture images of a 
meal being consumed to be transferred for further analysis [19,20]. As with the food monitor using shopping receipts [16], this type of monitoring still presents a burden to the subject under evaluation that might change an ad libitum eating behavior. A wearable device almost completely passive to the subject and still under development is proposed in [21] with a camera collecting images of the meal and a dietician later evaluating the image sequence to determine the food items.

The methodology we propose here is based on the use of features extracted from the monitoring of swallowing and chewing to segment a meal into the different food items it consists of. We believe that monitoring of chews and swallows over time provide a useful measure for food intake pattern identification of different categories of food since both activities are directly related to the food intake process in healthy humans.

\section{Methods}

\subsection{Data collection}

The data for the development of the proposed models described in this work were collected in a study reported in previous published work [8]. Data from 17 human subjects (11 males, 6 females) were used. The average body mass index across the sample population was equal to $28.81 \pm 6.61 \mathrm{~kg} / \mathrm{m}^{2}$, age of subjects varied from 18 to 48 years old. The subjects reported no dental problems that would deficit their normal masticatory ability to process food. An attempt to recruit a diverse population was made in terms of gender, ethnicity, age, and body size. Institutional Review Board approval was obtained for this study. Subjects read and signed the informed consent form document. Each subject performed from two to four meal experiments on separate days. In each experiment, subjects were asked to eat a meal consisting of a slice of cheese pizza, a can of $1 \%$ fat yogurt, an apple, a peanut butter sandwich (PB) and a cup of water consumed in order. The selection of foods represented different food properties that may impact chewing and swallowing, such as crispiness, moistness, softness/hardness and tackiness, and focused the experiment on inter-subject variability of chewing and swallowing pattern rather than inter-food variability. In half of the meal experiments, subjects were asked to remain silent, and in the other half they were involved into conversation with the experiment's operator.

During a meal, the subjects were monitored by a number of sensors capturing and characterizing the process of chewing and swallowing. Chewing was monitored by a strain sensor located in the area immediately below the ear. Swallowing was monitored by a microphone located over the laryngopharynx. In addition to these sensors the subjects were also videotaped in profile. Time-synchronous recordings from the sensors and video were reviewed in specially designed software and used for manual scoring process [8].

The produced manual scores contain timetable and duration of each swallow and chew as well as the number of chews in each mastication period [8]. Manual scores of the swallows and chews provided the initial data for the meal segmentation models. In total, 66 meals were scored, with around $17.5 \mathrm{~h}$ of meal recordings containing 5634 swallows and 44,038 chews.

\subsection{Features extraction}

Information from swallowing and chewing scores was analyzed to extract features which were used for meal segmentation into different food items. Three types of features were defined that characterize the timing and frequency of chewing and swallowing. The first feature analyzed was the location in time $t_{i}$ of each swallow for $i=2, \ldots, k$ total number of swallows in a particular subject's meal. This feature was obtained as the center point between the beginning and end, defined by the manual scores, of the th swallow. The 
underlying assumption for using this feature is that most individuals consume foods bite by bite at a time during a meal, thus making occurrence time of each swallow contained in each bite an important feature in grouping the swallows related to a certain food.

The second feature was the Time to Preceding Swallow for the $i$ th swallow expressed in seconds and defined as $T P S_{i}=t_{i}-t_{i-1}$, which is the difference in occurrence time between a swallow located at moment $t_{i}$ with respect to the previous swallow located at moment $t_{i-1}$. The third feature was the number of chews between two consecutive swallows, $t_{i}$ and $t_{i-1}$, defined as Chews Preceding a Swallow $C P S_{i}=\left.N_{i}\right|_{i_{i-1}} ^{t_{i}}$.

Although eating behavior is influenced by a large number of external factors like palatability, time of day, day of the week, social environment, etc. [22], the main hypothesis of the experiment was that different foods generate different chew and swallow sequences due to different properties of the foods being consumed in a meal [23]. Fig. 1 shows an example of the TPS and CPS signals generated for a complete meal of one subject. It can be seen that different foods in the experimental meal create distinct patterns in terms of TPS and CPS metrics over time. As an example, moist semi-solid food (yogurt) exhibits low chewing duration and high frequency of swallowing, while a tacky food (peanut butter sandwich) exhibits low swallowing frequency with prolonged periods of chewing. Thus, transitions between different foods in a meal can potentially be discovered by monitoring changes in features of the time sequence of chews and swallows.

Each swallow during consumption of given food was assigned a class label. Class labels were defined as $C_{i} \in\{1,2,3,4,5\}$, representing swallows of pizza, yogurt, apple, PB and water respectively. Feature vectors $T_{i}$ were built based on different combinations of the described features as:

$$
T_{i}=\left.\left\{x_{i}, C_{i}\right\}\right|_{i=1} ^{k}
$$

where the combination of features for different space dimensionality was studied to observe the impact of $t_{i}$, TPS and CPS, extracted from the data, in the performance of the implemented models. Each of these feature combinations were defined as:

- $x_{i} \in \Re^{1}=\left\{t_{i}\right\}$

- $x_{i} \in \Re^{2}=\left\{C P S_{i}, T P S_{i}\right\}$

- $x_{i} \in \Re^{3}=\left\{t_{i}, C P S_{i}, T P S_{i}\right\}$

- $x_{i} \in \Re^{7}=\left\{t_{i}, T P S_{i-1}, C P S_{i-1}, T P S_{i}, C P S_{i}, T P S_{i+1}, C P S_{i+1}\right\}$,

for $i=1,2, \ldots, k$ and $k$ being the total number of swallows in a subject's meal. The first feature set $\left(\Re^{1}\right)$ investigated the effect of sequence in food consumption. The second feature set $\left(\Re^{2}\right)$ focused just on properties of chewing and swallowing associated with a given swallow, regardless of where they reside in time sequence of food intake. The third $\left(\Re^{3}\right)$ and fourth feature $\left(\Re^{7}\right)$ sets studied if the accuracy of meal segmentation can be further improved by combining information from $\left(\Re^{1}\right)$ and $\left(\Re^{2}\right)$, and introducing information from the nearest swallows, respectively.

\subsection{Meal segmentation}

In order to identify groups of food within a meal, a clustering approach was used based on two different techniques. The first one is a relatively new unsupervised method named Affinity Propagation (AP); this method is a clustering algorithm that passes messages between data points to identify exemplars and form clusters around them [24]. It operates by 
simultaneously considering all data points as potential exemplars and exchanging messages between data points until a good set of exemplars and clusters emerges. One of the advantages of AP over other clustering techniques is the ability to determine the number of clusters from a given data set [24].

This approach was used in an attempt to automatically estimate the number of segments within the meal used in our experiments without specifying any a priori information about the number of food items consumed by the subjects using the feature vectors $T_{i} \in \Re^{d}$ as defined in Section 2.2.

The accuracy of the AP model in finding the correct number of food items was obtained based on an error measure across all subjects and all experiments as:

$$
\text { Acc_cluster\% }=(1-E) \times 100
$$

where $E$ is the error measure of finding exactly 5 known food items, defined as $E=|n-5| / 5$, with $n$ being the number of estimated food items. Additionally, to compare accuracy between supervised and unsupervised approaches, a second model was implemented using Agglomerative Hierarchical Clustering (AHC) [25-27]. First, each feature vector $T_{i} \in \Re^{d}$ was considered a singleton cluster and the proximity matrix was calculated for the $k$ initial clusters, using unweighted average distance algorithm (UGPGMA) with Euclidian distance as a measure between points [27]. This linkage distance represents the extent in which clusters are proximal to each other. A threshold $a$ was set in such a way that clusters with linkage distances lower than a certain value were merged into a new cluster. Representation of the linkage distances can be observed at the dendrogram shown in Fig. 2. Here, the linkage distance is normalized to range $[0,1]$.

With an $a=0$, each swallow represented by a leaf on the dendrogram is interpreted as a cluster and, consequently, as a different food item. At the other extreme, with an $a=1$ all swallows will be merged into one cluster, representing only one food item.

To find an optimal threshold $a$, half of the subjects were selected randomly to be used as the training data set, and the remaining subjects were used as the validation data set. For each meal in the training set, all possible $a$ values that give 5 groups were used to build a probability density estimation (PDE).

Fig. 3 shows the distribution of all possible $a$ values obtained from the training data set. The optimal $a$ selected is the one with the highest probability of this obtained distribution. This $a$ value is then applied as the threshold for the normalized linkage distances of each meal in the validation set.

In Fig. 2, the optimal $a$ obtained after training is shown as the dash line that partitions the dendrogram, estimating in the given example five different food items.

The accuracy of the correct number of food items was calculated for the validation data set based on the error measure, as in Eq. (2).

Ten runs were performed using hold-out cross validation to reduce variability due to random selection of the subjects for the training data set, and overall accuracy was assessed as the average across these runs.

\subsection{Classification of swallows}

The capability of the models described above to classify swallows into groups corresponding to food items consumed separately by a subject was analyzed, thus evaluating 
the predictive power of features derived from chewing and swallowing in the meal segmentation. The association of swallows into different group types, 5 food items for the particular case of this study, is of significant importance for the development of our proposed MIB methodology, since it has been observed that bite size, hence swallow size, differs for different types of food, i.e. solids and semi-liquids [23], and that the number of swallows is proportional to consumed food mass [10].

Being able to classify swallows into groups of different foods may represent a viable measure for mass estimation of total food consumed.

Fig. 4(a) displays an example of $T_{i}=\left.\left\{x_{i} C_{i}\right\}\right|_{i=1} ^{k}$ for $x_{i} \in \Re^{3}$. It can be observed how each one of the swallows was grouped into one of the five clusters for this particular experiment using the AP model. Fig. 4(b) shows the projection of the clusters into the CPS-Time plane and Fig. 4(c) shows the projection into the TPS-Time plane, where separation of different clusters can be observed. The projection of the clusters into the plane CPS-TPS is shown in Fig. 4(d), showing no obvious separation of different clusters.

In order to evaluate the ability of the segmentation models to associate all swallows correctly into their corresponding food item, an accuracy measure was defined as the ratio of correctly classified swallows $P$ for a given food type class $C$ over the real number of swallows $R$ belonging to that same class:

$$
A c c_{-} S w_{j C} \%=\frac{P_{j C}}{R_{j C}} \times 100
$$

for $j=1, \ldots, 5$ number of total subject's consumed food items.

\section{Results}

Meal experiments from 17 subjects were used to analyze two different models to automatically perform meal segmentation. Swallow timing, TPS and CPS signals extracted from swallows and chews occurrence respectively were used as features for these two models.

The first model was based on AP. Using this technique, the number of exemplars emerged from the message-passing procedure [24], that is, AP was capable of finding the optimal number of exemplars of the data set without any a priori knowledge. The second model was a supervised method based on AHC that sets a threshold and merges clusters with low linkage distances.

Table 1 shows the accuracy results (from Eq. (2)) as the average and standard deviation (SD) across all subjects of the different combinations of features described in Section 2.2 for both AP and AHC.

Fig. 5 shows the distribution comparison between the two models, AP and AHC, for automatically finding the number of food items in a meal. Results are expressed as a percentage across all subjects for the best case obtained of each model, that is, $T_{i} \in \Re^{7}$ and optimal threshold $a=0.286$ for the AHC model, which presents a significant increase from $91.3 \%$ to $95.3 \%$ (paired $t$-test, $p<0.0015$ ).

In comparison of obtained meal segmentation, accuracies for visits where subjects remained silent during the meal with those where subjects were encouraged to participate in conversation no significant difference was observed (paired $t$-test, $p>0.05$ ). 
Accuracy of the classification of swallows (from Eq. (3)) is shown in Fig. 6, where both models represent the correct association of swallows into each food type.

\section{Discussion}

This study presents two different models to automatically obtain the number of food items involved in a subject's meal. To our knowledge, this is the first attempt to automatically perform such segmentation which is of interest for studies of dietary activities, obesity and other eating disorders.

When performing automatic meal segmentation, AP found a range of 4-7 (Fig. 5) food items for a 5-food items meal with an accuracy of correct segmentation of 90.3\% (Table 1). The supervised AHC model implemented found a range of 4-6 food items (Fig. 5) with 95.3\% accuracy (Table 1) with training performed on half of the dataset with the rest of the dataset used for validation. These results show that the supervised model based on AHC has a higher performance compared to the AP model in segmenting a meal, which can be seen in the $75.76 \%$ of finding exactly 5 food items within the meal, compared to the $59 \%$ of the AP model (Fig. 5). This might be explained by the ability of the supervised learning methods to adjust the tradeoff between the bias (incorrect output prediction) and the variance (different output for different training sets) or the learned model [28].

Swallow location in time $t_{i}$ appears to be the most efficient individual feature used by the implemented models. This is expected as the meals are typically consumed in a specific sequence (e.g. salad, entree, desert) and many consecutive chews and swallows will belong to the same food type and grouped in time. However, the accuracy is improved $>4 \%$ by introducing the CPS and TPS features into the models and using $\Re^{7}$ neighbors results in the AHC model (Table 1). The highest accuracy obtained with the supervised model with a feature combination of $x_{i} \in \Re^{7}$ suggests that it is not only timing of the swallows which drives the clustering, but significant contribution is provided by CPS and TPS features characterizing chewing and swallowing process.

When performing the classification of swallows of different food items with a priori knowledge of the number of food items, AHC and AP achieved very similar accuracies (Fig. 6). Association of swallows into groups may result in an accurate mean to measure the total consumed mass of a subject, due to the constant bite size within similar foods [23], and the significant correlation between the number of swallows and the mass ingested by subject [10]. Water swallow classification resulted in the highest accuracy for both methods. The high frequency of swallowing in liquids, which results in lower TPS values, but particularly in the zero values of the CPS due to the absence of chewing, may explain why the highest accuracy was obtained in this case.

Also, fairly good accuracy was obtained for pizza and yogurt, since the transition from one to the other results in significant changes in the TPS and CPS features. On the other hand, distinction between apple and peanut butter swallows resulted in a lower accuracy which can be explained by similarity of their TPS and CPS characteristics across all subjects.

The high accuracy evidence for efficiently separating swallows of pizza from yogurt, and peanut butter from water, suggests that the methodologies described in this study may be used in more general cases under free-living conditions where differences between segments within a meal are significant. Additionally, the significant differences in the TPS and CPS signals may result useful in building models that identify the types of foods consumed, i.e. solid, semi-solid and/or liquid intake within a meal. 
The features $t_{i}$, TPS and CPS used in this study were obtained from manual scores of sound recordings of swallows and strain sensor signal of chews, but they may potentially be obtained from automatic scores of swallowing and chewing detection methods that would enable creation of a wearable device [11]. This is part of our ongoing research goals.

One limitation of the reported study is that food items were consumed unmixed and in a specific order, which allowed to eliminate uncertainty in inter-food variation of food properties. In free-living conditions, the number and type of food items within a meal will vary according to subject's behavior and preferences [29]. Further study is needed to test the clustering algorithms performance on unrestricted meals. However, the presented clustering approaches should be directly applicable to unrestricted meals as food intake is cumulative over time [30] and consumption normally happens sequentially in a bite-by-bite manner, similar to the meal in this study.

The proposed methodology is a first step toward the automatic meal segmentation that could eventually be used in the study of eating disorders, obesity, and in biofeedback applications aimed at managing and monitoring ingestive behavior with unconstraint conditions, i.e. time of food intake, number of food types consumed and the order of consumption.

\section{Conclusion}

Two models for automatic meal segmentation into distinct food items were proposed and evaluated on data collected from 17 subjects. An overall accuracy of $>95 \%$ was obtained when estimating the number of food items in a meal using the implemented model based on supervised Agglomerative Hierarchical Clustering, compared to a $90 \%$ of the unsupervised Affinity Propagation model. Experimental results suggest that these features may be used as predictors of the composition of food items within a meal. The proposed models may be advantageous for development of an accurate, inexpensive, and non-intrusive methodology for objective monitoring of food intake in free living conditions.

\section{Acknowledgments}

The project described was supported by Grant Number R21DK085462 from the National Institute of Diabetes and Digestive and Kidney Diseases. The content is solely the responsibility of the authors and does not necessarily represent the official views of the National Institute of Diabetes and Digestive and Kidney Diseases or the National Institutes of Health.

\section{References}

1. Livingstone MBE, Black AE. Markers of the validity of reported energy intake. The Journal of Nutrition. Mar 3.2003 133:895S-920S. [PubMed: 12612176]

2. Champagne CM, et al. Energy intake and energy expenditure: a controlled study comparing dietitians and non-dietitians. Journal of the American Dietetic Association. Oct 10.2002 102:14281432. [PubMed: 12396160]

3. Day N, McKeown N, Wong M, Welch A, Bingham S. Epidemiological assessment of diet: a comparison of a 7-day diary with a food frequency questionnaire using urinary markers of nitrogen, potassium and sodium. International Journal of Epidemiology. Apr 2.2001 30:309-317. [PubMed: 11369735]

4. Wrieden, W.; Peace, H.; Armstrong, J.; Barton, K. Short review of dietary assessment methods used in National and Scottish Research Studies. Working Group on Monitoring Scottish Dietary Targets Workshop; 2003 September;

5. Bertéus Forslund H, Torgerson JS, Sjöström L, Lindroos AK. Snacking frequency in relation to energy intake and food choices in obese men and women compared to a reference population. International Journal of Obesity. Jun 6.2005 29:711-719. [PubMed: 15809664] 
6. Wansink B, Payne CR, Shimizu M. 'Is this a meal or snack?' Situational cues that drive perceptions. Appetite. Feb 1.2010 54:214-216. [PubMed: 19808071]

7. Lafay L, Mennen L, Basedevant A, Charles MA, Borys JM, Eschwège E, Romon M. Does energy intake underreporting involve all kinds of food or only specific food items? Results from the Fleurbaix Laventie Ville Santé (FLVS) study. International Journal of Obesity and Related Metabolic Disorders: Journal of the International Association for the Study of Obesity. Nov 11.2000 24:1500-1506. [PubMed: 11126348]

8. Sazonov E, Schuckers S, Lopez-Meyer P, Makeyev O, Sazonova N, Melanson EL, Neuman MR. Non-invasive monitoring of chewing and swallowing for objective quantification of ingestive behavior. Physiological Measurement. May 5.2008 29:525-541. [PubMed: 18427161]

9. Sazonov E, Schuckers S, Lopez-Meyer P, Makeyev O, Sazonova N, Melanson EL, Neuman MR. Reply to "Comment on "Non-invasive monitoring of chewing and swallowing for objective quantification of ingestive behavior. Physiological Measurement. 2009 May.30:L5-L7.

10. Sazonov E, Schuckers S, Lopez-Meyer P, Makeyev O, Melanson EL, Neuman MR, Hill JO. Toward objective monitoring of ingestive behavior in free-living population. Obesity. May 10.2009 17:1971-1975. [PubMed: 19444225]

11. Sazonov ES, Makeyev O, Schuckers S, Lopez-Meyer P, Melanson EL, Neuman MR. Automatic detection of swallowing events by acoustical means for applications of monitoring of ingestive behavior. IEEE Transactions on Biomedical Engineering. 2010; 57(3):626-633. [PubMed: 19789095]

12. Lopez-Meyer P, Makeyev O, Schuckers S, Melanson EL, Neuman MR, Sazonov E. Detection of food intake from swallowing sequences by supervised and unsupervised methods. Annals of Biomedical Engineering. Mar 8.2010 38:2766-2774. [PubMed: 20352335]

13. Pike KM. Long-term course of anorexia nervosa: response, relapse, remission, and recovery. Clinical Psychology Review. Jun 4.1998 18:447-475. [PubMed: 9638357]

14. Schebendach JE, Mayer LES, Devlin MJ, Attia E, Contento IR, Wolf RL, Walsh BT. Dietary energy density and diet variety as predictors of outcome in anorexia nervosa. The American Journal of Clinical Nutrition. Apr 4.2008 87:810-816. [PubMed: 18400701]

15. Wing RR, Marcus MD, Salata R, Epstein LH, Miaskiewicz S, Blair EH. Effects of a very-lowcalorie diet on long-term glycemic control in obese type 2 diabetic subjects. Archives of Internal Medicine. Jul 7.1991 151:1334-1340. [PubMed: 2064484]

16. Mankoff, J.; Hsieh, G.; Hung, HC.; Lee, S.; Nitao, E. Using low-cost sensing to support nutritional awareness. Proceedings of the 4th International Conference on Ubiquitous Computing; London, UK. 2002. p. 371-376.

17. Chang, K.; Liu, S.; Chu, H.; Hsu, J.; Chen, C.; Lin, T.; Chen, C.; Huang, P.; Fishkin, K.; Schiele, B. The diet-aware dining table: observing dietary behaviors over a table-top surface Pervasive Computing. Vol. 3968. Springer; Berlin/Heidelberg: 2006. p. 366-382.

18. Amft O, Troster G. On-body sensing solutions for automatic dietary monitoring. Pervasive Computing, IEEE. 2009; 8(2):62-70.

19. Martin CK, Han H, Coulon SM, Allen HR, Champagne CM, Anton SD. A novel method to remotely measure food intake of free-living people in real-time. The British Journal of Nutrition. Mar 3.2009 101:446-456. [PubMed: 18616837]

20. Six BL, Schap TE, Zhu FM, Mariappan A, Bosch M, Delp EJ, Ebert DS, Kerr DA, Boushey CJ. Evidence-based development of a mobile telephone food record. Journal of the American Dietetic Association. Jan 1.2010 110:74-79. [PubMed: 20102830]

21. Sun M, Fernstrom JD, Jia W, Hackworth SA, Yao N, Li Y, Li C, Fernstrom MH, Sclabassi RJ. A wearable electronic system for objective dietary assessment. Journal of the American Dietetic Association. Jan 1.2010 110:45. [PubMed: 20102825]

22. de Castro JM. Eating behavior: lessons from the real world of humans. Nutrition (Burbank, Los Angeles County, CA). Oct 10.2000 16:800-813.

23. Bellisle F, Guy-Grand B, Le Magnen J. Chewing and swallowing as indices of the stimulation to eat during meals in humans: effects revealed by the edogram method and video recordings. Neuroscience \& Biobehavioral Reviews. Mar 2.2000 24:223-228. [PubMed: 10714385] 
24. Frey BJ, Dueck D. Clustering by passing messages between data points. Science. Feb 5814.2007 315:972-976. [PubMed: 17218491]

25. Alpaydin, E. Introduction to Machine Learning. MIT Press; 2004.

26. Duda, RO.; Hart, PE.; Stork, DG. Pattern Classification. Vol. 24. John Wiley \& Sons; New York: 2001.

27. Xu, R.; Wunsch, DC. Clustering. John Wiley and Sons; 2009.

28. James GM. Variance and bias for general loss functions. Machine Learning. May; 2003 51(2):115135.

29. Schwerin HS, Stanton JL, Smith JL, Riley AM Jr, Brett BE. Food, eating habits, and health: a further examination of the relationship between food eating patterns and nutritional health. The American Journal of Clinical Nutrition. May 5.1982 35:1319-1325. [PubMed: 7081115]

30. Westerterp-Plantenga MS. Eating behavior in humans, characterized by cumulative food intake curves—a review. Neuroscience \& Biobehavioral Reviews. Mar 2.2000 24:239-248. [PubMed: 10714387] 

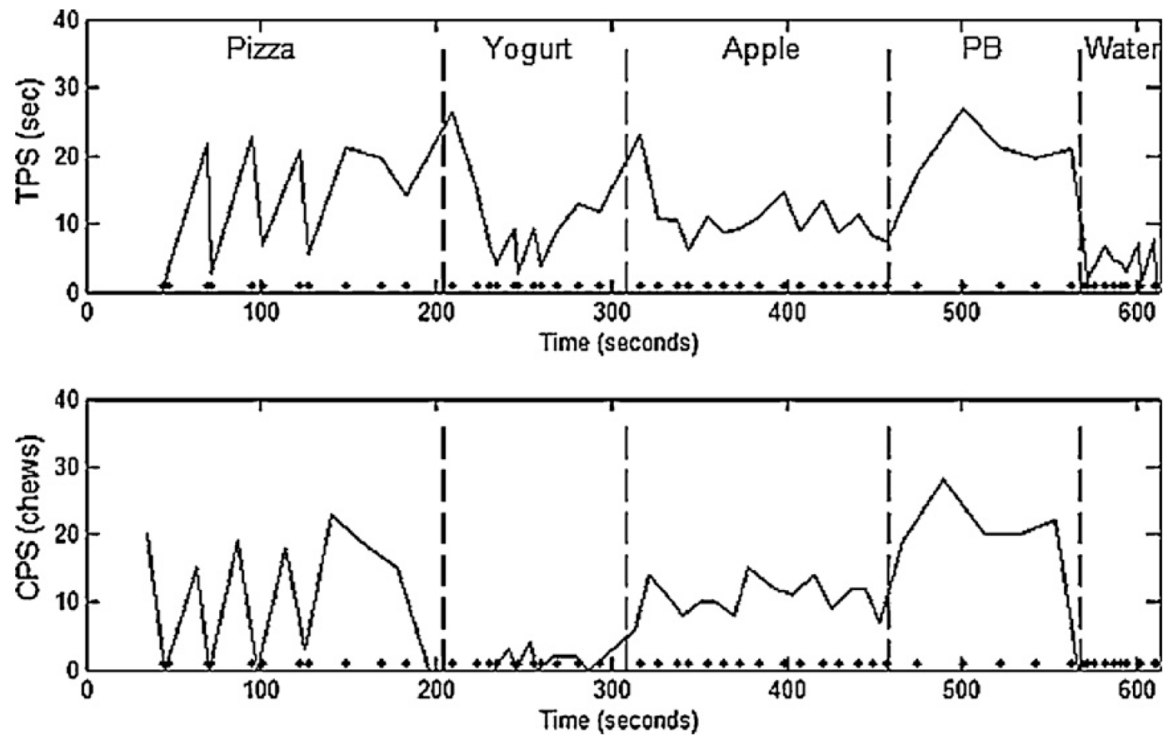

Fig. 1.

Example of a complete meal of a subject. The figure on top shows the TPS signal, and the one on the bottom shows the CPS signal. Vertical dashed lines represent the time boundaries of the different segments or food items. Swallow locations in time are represented as dot marks. 


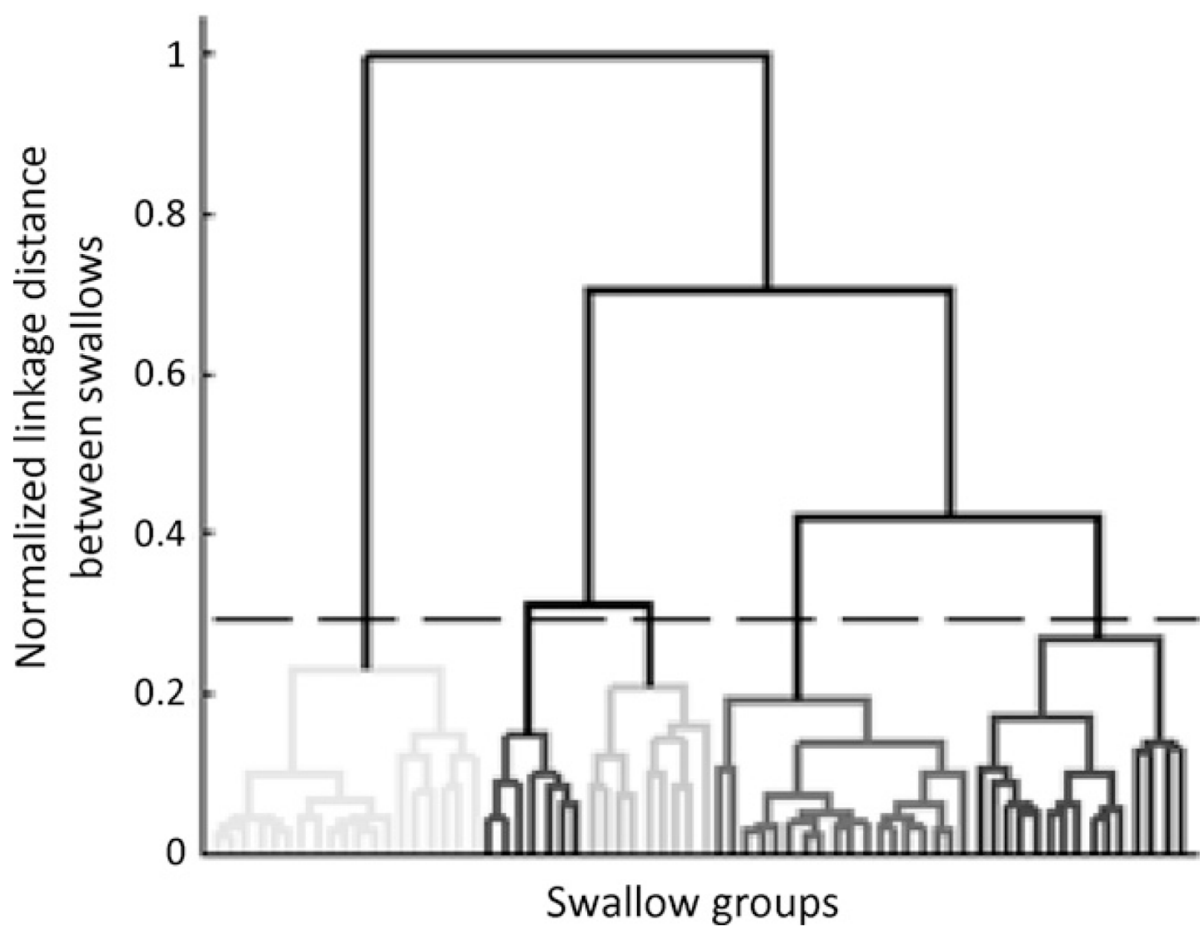

Fig. 2.

The dendrogram shows the hierarchical tree for all swallows with an optimal threshold $a$ (dashed line) obtained using supervised learning to find the correct number of food items, described by the branches below the threshold. 


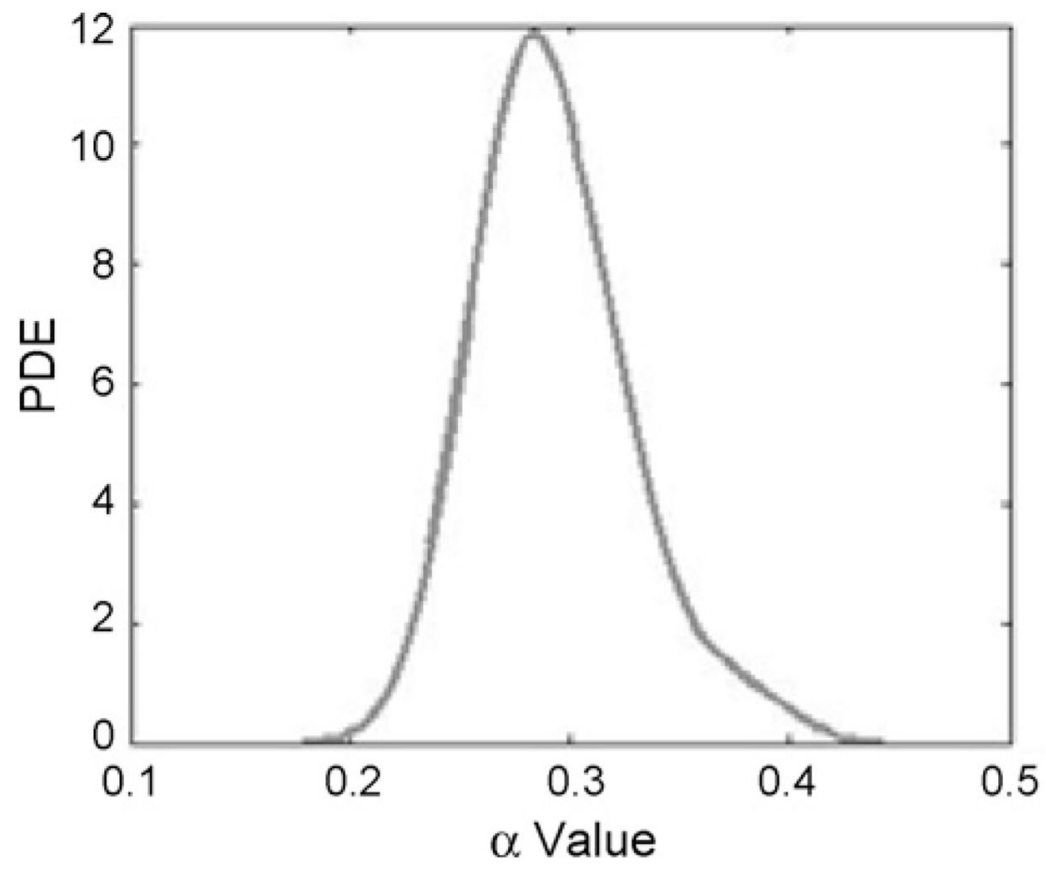

Fig. 3.

Probability density estimation of $a$ obtained using the training set. The $a$ with the highest probability represents the optimal threshold of the linkage distances to group swallows into groups of the same food type. 

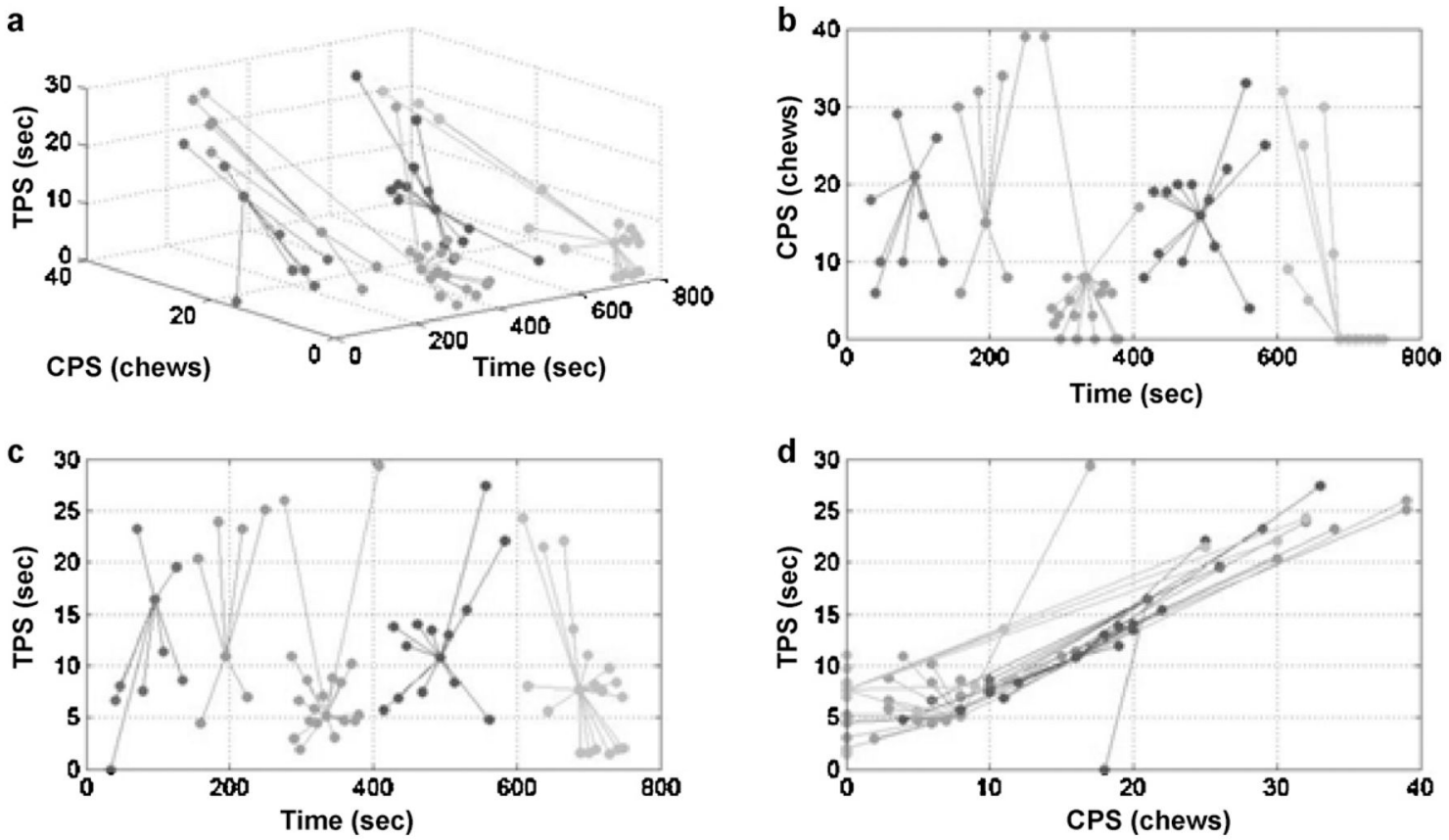

Fig. 4.

Grouping of swallows using the Affinity Propagation clustering method. In increasing order of time, the swallow groups belong to: pizza, yogurt, apple, PB and water. 


\section{- Hie rarchical Affinity Propagation}

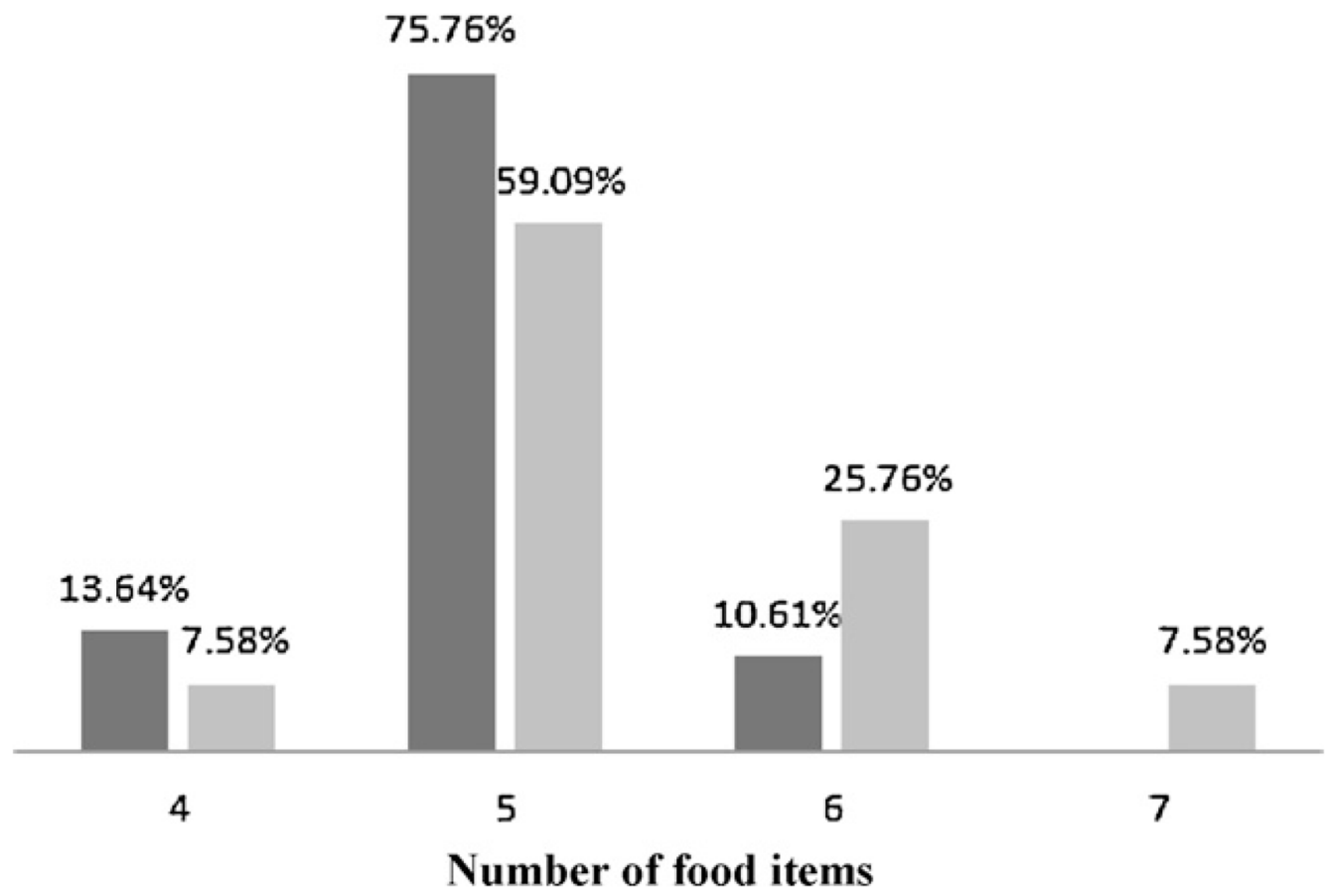

Fig. 5.

Automatic meal segmentation of food items consumed in a meal using Affinity Propagation and Agglomerative Hierarchical Clustering. Results display the percentage of estimated number of food items over the a priori known number of 5 food items using $T_{i} \in \Re^{7}$ and an optimal threshold $a=0.286$ for the AHC model. 


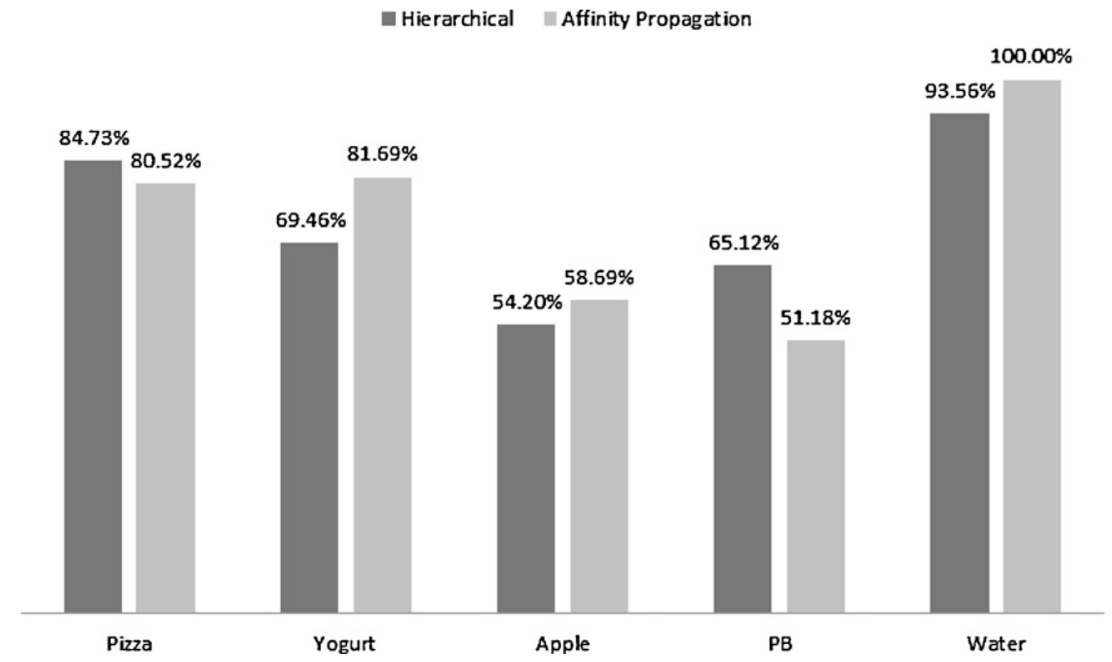

Fig. 6.

Results obtained on the classification of swallows from the Affinity Propagation and the Agglomerative Hierarchical Clustering algorithm with specified 5 clusters. 


\section{Table 1}

Average accuracy and standard deviation of the models implemented on finding the correct number of 5 food items for different combinations of features.

\begin{tabular}{|c|c|c|c|c|}
\hline & $x_{i} \in \Re^{1}$ & $x_{i} \in \Re^{2}$ & $x_{i} \in \Re^{3}$ & $x_{i} \in \Re^{7}$ \\
\hline $\mathrm{AP}$ & $89.7 \%$ (SD 13.2) & $21.8 \%(\mathrm{SD} 40.2)$ & $88.8 \%($ SD 14.1$)$ & $90.3 \%$ (SD 12.7) \\
\hline $\mathrm{AHC}$ & $91.3 \%$ (SD 9.9) & $70.7 \%$ (SD 29.9) & $92.9 \%(\mathrm{SD} 9.6)$ & $95.3 \%(\mathrm{SD} 8.5)$ \\
\hline
\end{tabular}

